\title{
Currículo por competencias visto desde la educación inclusiva: desafío que responde a los factores de exclusión e inequidad social
}

\author{
Curriculum by competencies seen from inclusive education: a \\ challenge that responds to the factors of exclusion and social \\ inequity
}

Franklin Manuel Osorio Galindo

Universidad Metropolitana de Educación Ciencia y Tecnología. Ciudad de Panamá, Panamá

Recibido: 05/08/2021

Revisado: 20/09/2021

Aceptado: 27/09/2021

Publicado: $31 / 10 / 2021$

\section{RESUMEN}

El presente artículo de reflexión, surge desde el espacio académico del módulo desarrollo de competencias mediante el planeamiento didáctico del Doctorado en Ciencias de la Educación de la Universidad Metropolitana de Educación Ciencia y Tecnología UMECIT (Panamá), el cual tiene por propósito visualizar los aportes del enfoque por competencias al diseño curricular, las prácticas de enseñanza y las formas de evaluación que tienen lugar en la educación. La metodología desarrollada fue la cualitativa-reflexiva, utilizando fuentes bibliográficas o documentales, los principales hallazgos están enfocados hacia la explicación, posturas conceptuales e importancia de la educación inclusiva como modelo educativo integrador y cómo responde a los desafíos de exclusión e inequidad social. Igualmente, resalta elementos transformacionales de la escuela como espacio incluyente, permeado en equidad a través del currículo. A modo de conclusión de esta reflexión teórica, se reconocen las estrategias y rediseños curriculares que propenden al desarrollo de las competencias y aprendizajes de los estudiantes, donde se incluyan a todos desde un ambiente escolar. No solamente con el acceso se atiende a los requerimientos en inclusión, también es necesario la equidad en la prestación.

Palabras claves: Educación inclusiva, equidad, acceso, currículo, competencias.

\section{ABSTRACT}

This article for reflection arises from the academic space of the competence development module through the didactic planning of the Doctorate in Educational Sciences of the Metropolitan University of Education, Science and Technology UMECIT (Panamá), which aims to visualize the contributions of the Competency-based approach to curriculum design, teaching practices, and forms of evaluation that take place in education. The methodology developed was quali- 
tative-reflective, using bibliographic or documentary sources, the main findings are focused on the explanation, conceptual position and importance of inclusive education as an inclusive educational model and how it responds to the challenges of exclusion and social inequity. It also highlights elements transformations of the school as an inclusive space, permeated in equity through the curriculum. By way of conclusion of this theoretical reflection, the strategies and curricular redesigns that tend to the development of the competences and learning of the students are recognized, where everyone is included from a school environment. Not only with access is it attended to the inclusion requirements, it is also necessary to provide equity.

Keywords: Inclusive education, equity, access, curriculum, competencies.

\section{INTRODUCCIÓN}

En los últimos años, los gobiernos han incluido programas y reformas que permean el sistema educativo para dar respuesta a dos desafíos y factores sociales: la inclusión y la equidad. Los aportes conceptuales y sociales de la educación inclusiva fortalecen la consecución de los fines educativos en la atención de los diferentes grupos poblacionales, es decir, favorecen en la atención a la diversidad, bajo un enfoque de derechos, desde un piso curricular flexible y diversificado visibilizados desde la equidad. Este es reafirmado por Martín (2013) quien afirma que "La educación inclusiva figura en este momento en las agendas de todos los organismos internacionales educativos, pero, lo que es más importante, ha comenzado a ser un valor asumido por una mayoría de la sociedad" (p. 9).

A través de los debates, discursos y encuentros en educación, ha permitido repensar la inclusión como una oportunidad para replantear el sistema educativo, para Rambla (2012) "la educación inclusiva se deriva de cuatro maneras básicas de entenderla, como: un derecho humano, la atención prioritaria a grupos categorizados NE, la atención a grupos socialmente vulnerables $y$, como un eje transformacional del sistema educativo en su conjunto" (p. 39). Igualmente, la OEI (2010) citado por Castillo \& Gamboa (2017) estable- ce que "es menester un sistema formativo que atienda las necesidades educativas especiales y específicas de los individuos, es decir, una educación inclusiva para todos" (p. 8).

En busca de un cambio transformacional del sistema, la educación inclusiva como modelo educativo integrador, responde a los desafíos de exclusión e inequidad social y destaca la responsabilidad de los gobiernos, en implementarlo como eje transversal en el sistema educativo y la escuela como espacio incluyente permeado en equidad a través del currículo. En este sentido se permea, la inserción social (acceso al sistema educativo) y realización personal de los niños, niñas y jóvenes, que atienda las características individuales, sus motivaciones y permita el desarrollo de competencias desde su significación y flexibilidad (equidad).

Ante estas promesas, la escuela se plantea los interrogantes ¿Cómo responder ante la diversidad y cómo hacer para que todos y todas se sientan identificados e incluidos en un sistema educativo que les permita crecer aún fuera de la escuela? ¿Cómo alcanzar una educación de calidad donde se reconozca la equidad?

La educación inclusiva: desafío que responde a los factores de exclusión e inequidad social.

La necesidad de responder a los anteriores interrogantes y los múltiples desafíos 
que se presentan en la educación y la sociedad, ha propiciado la reflexión y los acuerdos internacionales, debates y propuestas sobre cómo mejorar los procesos educativos, cómo diseñar las estrategias y metodologías para la enseñanza afines a una filosofía de la educación centrada en la formación integral, entendiendo al educando como un ser diverso, que tiene sus formas propias de aprender y que se encuentra en múltiples contextos sociales, que tiene características propias y, por tanto, son muchas las diferencias entre los estudiantes que concurren a la escuela. Sumado a lo anterior, están los problemas por los que atraviesa la sociedad, como los conflictos políticos, los desplazamientos forzados, migraciones, la violencia intrafamiliar, la exclusión y la inequidad social, entre otros.

En concordancia con lo anterior, y a partir de la promulgación de los acuerdos en Marco de Acción de Dakar, Senegal 2000 "Educación para Todos: cumplir nuestros compromisos comunes", desde una visión de la inclusión y la equidad, se reflexiona sobre estas tendencias y desafíos. Así mismo, para Castillo \& Gamboa (2017), es importante que "los sistemas educativos aporten a la creación de las condiciones que garanticen el acceso de todos los individuos a una educación de calidad, inclusiva, multicultural y que fomente la diversidad y la democracia" (p. 9)

Este compromiso, fue ratificado en la declaración de educación para todos: Un asunto de derechos humanos (UNESCO, 2007). Este documento es el soporte para las deliberaciones de la Segunda Reunión Intergubernamental de Ministros de Educación del Proyecto Regional de Educación para América Latina y el Caribe (PRELAC), realizada en marzo de 2007 en Buenos Aires, Argentina. El tema central es la educación de calidad para todos, entendida como un bien público y un derecho humano fundamental que los Estados tienen la obligación de respetar, promover y proteger, con el fin de asegurar la igualdad de oportunidades en el acceso al conocimiento de toda la población. La agenda 2030 para el desarrollo sostenible, compromete a los Estados a través de su política educativa interna propendan a "garantizar una educación inclusiva y equitativa de calidad y promover oportunidades de aprendizaje permanente para todas y todos" (ODS 4), con la apropiación y apoyo a través de las políticas nacionales, se consideran como la piedra angular para una ejecución exitosa.

Desde una perspectiva general, la educación inclusiva como modelo educativo promueve la participación y el aprendizaje de todos y todas, indistintamente de sus características individuales, respuesta que debe atenderse desde las instituciones y el currículo. Cualquier niño o niña es educable en la escuela ordinaria, y se asocia con una educación de calidad para todos y todas sin excepciones. Por tanto, la diversidad es un valor en alza que cohesiona al grupo y que ofrece mayores posibilidades de aprendizaje. Para ello se plantean medidas con el objetivo de dar una respuesta educativa inclusiva, desde la organización escolar, como desde el currículo, los métodos y estrategias de enseñanza, acompañado desde el desarrollo profesional, innovación y la gestión escolar.

Sin embargo, uno de los mayores desafíos para la atención a la diversidad desde el currículo es la migración, este factor social afecta el sistema educativo. El desplazamiento de la población aumenta en las ciudades, incrementando la diversidad cultural en las aulas, puesto que, es la escuela la llamada en comulgar con estas necesidades a través 
de transformaciones administrativas, curriculares y programáticas que apoyen a los estudiantes que llegan desde las distintas partes del mundo o desde el interior del país.

En consecuencia, no es posible comprender las escuelas fuera del contexto de las influencias sociales y culturales. En estudios recientes por la OCDE (2019), la movilidad internacional seguirá aumentando. Como muestra de esto, señala que el número total de migrantes internacionales ha crecido en un $69 \%$ entre 1990 y 2017, aumentando de 153 a 258 millones de personas. Por tanto, los sistemas educativos deben establecer medidas para integrar de mejor manera a estudiantes de origen diverso y ofrecerles una educación de calidad.

Por otra parte, la revista SEMANA (2019), en su informe visibiliza esta situación global indicando que hay mucha más gente migrando y Asia ha remplazado a Europa como el destino más popular para los migrantes. Esta movilidad trae la diversidad cultural, la energía y la ambición de los recién llegados.

Como aspecto alternativo, la transformación curricular se implica dentro de las propuestas y reformas educativas para dar respuesta a las dinámicas de reducción del analfabetismo y la inclusión escolar de las poblaciones vulnerables, un ejemplo de ello es la proyección de los gobiernos en impulsar modelos etnoeducativos. Según el informe de la SITEAL (2019) "destaca todos los programas específicos orientados a la inclusión de estudiantes indígenas y a aquellos con algún tipo de discapacidad. Ello implica la revisión de los marcos curriculares, las estrategias pedagógicas y el rediseño de las dinámicas institucionales" (p. 7).
Ante los anteriores desafíos, muchos has sido los esfuerzos de los Estados para incorporar en el sistema educativo programas que minimicen las desigualdades en acceso al sistema escolar en todos los niveles. Una tendencia ha sido extender la obligatoriedad de la educación en los niveles iniciales y básicos. La incorporación de modelos educativos flexibles como oportunidades para las poblaciones vulnerables, rural, dispersa, étnica, población mayor y población con discapacidad. Una característica fundamental de la educación inclusiva es minimizar la discriminación desde los sistemas educativos, es decir poder garantizar el derecho a la educación.

Para Opertti \& Belalcazar (2008) "la educación inclusiva constituye un motivo de preocupación universal en aumento que informa e interesa a los procesos de reforma educativa, tanto en las regiones desarrolladas como en aquellas en vía de desarrollo" (p. 151).

En la medida, que las reformas y programas con enfoque inclusivo permean el sistema educativo, y las poblaciones que antes no eran atendida por la escuela, ingresen a las aulas escolares, se hace más notoria la dificultad para el componente curricular, tener una propuesta que los cobije o atienda según las necesidades particulares. Es aquí donde lo invisible se hace visible, elemento de entrada para la flexibilización curricular. El sistema, la escuela y el currículo se deben reinventar. Para Guijosa (2019) "Los salones de clase dejarán de ser estáticos y sus elementos y diseño favorecerán la interacción entre alumnos y profesores" (p. 2).

Dentro de esta dinámica, se requiere de un currículo abierto y flexible que posibilite una vasta gama de estrategias, dentro 
de ellas se destacan las aulas colaborativas. Éstas favorecen el aprendizaje y el desarrollo de competencias en el marco de la educación inclusiva, ya sean desde la presencialidad o la virtualidad, dependiendo del acceso y de las herramientas tecnológicas.

Las aulas colaborativas son una de las tendencias didácticas actuales, puesto que promueven la integración y participación de todos sus miembros en el aprendizaje, permiten crear redes de apoyo, tutorías, monitorias y posibilita que estudiantes diversos participen activamente en distintas actividades curriculares. Para Stainback \& Stainback, (2001) "Las iniciativas de colaboración entre compañeros en sus diversas formas, puede convertirse rápidamente en norma dentro de una escuela, estas iniciativas son de carácter multidireccional que unidireccional y favorece las prácticas de aulas con enfoque inclusivo" (p. 153)

Desde un enfoque multidireccional, muchas escuelas van incorporando poco a poco las estrategias curriculares, que ofrecen a los estudiantes una forma de aprendizaje más personalizada y mejor relacionada con la experiencia. El uso cada vez más habitual de métodos como el de la enseñanza del lenguaje total, las actividades directas, el aprendizaje cooperativo y la enseñanza por temas y basados en proyectos, hace posible adaptarse a un conjunto de alumnos mucho más amplio, al tiempo que proporciona una experiencia más rica a todos los estudiantes.

\section{Lenguaje Total}

Según este enfoque, el relato, la literatura y la expresión escrita son fundamentales para la clase. Leer, escribir, hablar y escuchar no son actividades que se enseñen en clases aisladas, como conjuntos cerrados de destre- zas, sino que se consideran interconectados y se enseñan como partes naturales de cada clase. Este enfoque se centra en el niño y trata de sacar partido de los intereses y necesidades de cada uno. Los alumnos que reciben una enseñanza de lenguaje total, escriben realmente (en vez de practicar la escritura) y leen literatura (en vez de ser meros lectores).

\section{Micro sociedad o actividades directas.}

El objetivo consiste en dar a los alumnos una idea precisa del mundo real, enseñando cómo funcionan las instituciones mediante la participación, capacitando, a los alumnos para la adopción de papeles activos cuando sean adultos.

\section{Enfoque Cooperativo}

Los enfoques relacionados con el aprendizaje cooperativo están adquiriendo cada vez más popularidad, a medida que las escuelas prestan mayor atención al incremento del rendimiento de los alumnos, así como las habilidades sociales. El aprendizaje cooperativo enseña a los niños a trabajar con sus compañeros con el fin de alcanzar objetivos comunes, haciendo más fácil la inclusión de todos los alumnos como agentes activos en clase. Dadas las características de los enfoques cooperativos, los maestros pueden estructurar funciones adaptadas a las capacidades de los individuos.

\section{Enfoques temáticos - basados en proyectos}

Las expresiones temáticas y basadas en proyectos se utilizan a veces para describir actividades de aprendizaje que enfatizan la aplicación de las destrezas que van apareciendo en los niños, y pueden relacionarse directamente con la vida, las experiencias y los intereses de los estudiantes. Los proyectos consisten en estudios profundos de temas interesantes para los niños y jóvenes y pueden 
estructurarse de manera que los niños con características diversas puedan hacer aportaciones al esfuerzo del grupo. Las actividades de los proyectos pueden incluir investigaciones (uso de la biblioteca y otros recursos de información), tareas de gestión (funcionamiento de la tienda escolar), actividades de creación en interpretación de dramática. Como los alumnos no solo hablan y escriben, sino que "hacen", se incrementan en gran medida las oportunidades de participación activa de un individuo con necesidades educativas.

Estos enfoques se adaptan mucho mejor que las prácticas tradicionales de lecciones magistrales, trabajos en el pupitre y enseñanza basada en el libro de texto. Cuando el contenido del currículo se organiza en torno a una actividad más general y significativa, es posible estimular a los alumnos en diferentes niveles.

Del mismo modo, una de las herramientas que ha permitido el acceso y mayor cobertura en los niveles educativos para la población diversa en el marco de la educación inclusiva ha sido la tecnología. La televisión como medio de comunicación masiva ha incluido traductores en lenguajes de señas para los programas nacionales, y programas educativos. Igualmente, el uso de las tecnologías de la información y la comunicación (TIC) en las escuelas y los hogares, junto con programas de conectividad, han permitido el acceso a un mundo globalizado, incluyendo el de la información. Sin embargo, se requiere de mayor compromiso de los actores educativos, padres, estudiantes y docentes en el uso responsable de la tecnología para favorecer el sistema educativo. Según Guijosa (2019) argumenta que la tecnología ha tenido mayor influencia en las aulas, "lo que demandará mayor apoyo y compromiso por parte de las instituciones educativas, docentes y padres de familia respecto al uso que dan los estudiantes a las herramientas tecnológicas" (p. 2).

Igualmente, las tendencias curriculares con enfoque inclusivo permiten responder de alguna manera favorable los desafíos del sistema escolar en atender el acceso y equidad de las poblaciones marginales y a la vez estar al nivel de las exigencias internacionales. Para Correa (2011) citado por Osorio (2013), se podrían resaltar las siguientes tendencias curriculares: "La flexibilización e interdisciplinariedad del currículo, la globalización curricular, currículo y desarrollo humano integral, currículo e inclusión, currículo, ciudadanía y gobernabilidad social, entre otros" (p. 53).

Es importante resaltar que el acceso a la población diversa en los niveles educativo de carácter obligatorio, no radica únicamente en incluirlos en un aula escolar, sino en la transformación y aplicación de una vasta gama de estrategias curriculares que respondan a las necesidades de los estudiantes y la realidad de la sociedad, es decir, una igualdad de oferta en el sistema educativo. No solamente en el acceso se atienden los requerimientos, en equidad también es necesaria la igualdad en la prestación del servicio, es decir se deriva de dos factores. Para Martín (2013).

"El concepto de equidad ha evolucionado haciéndose progresivamente más exigente. Un primer nivel de equidad remite a la igualdad de acceso: todo alumno debe tener garantizada una plaza escolar. Pero esto no es suficiente, también se contribuye a la equidad cuando se garantiza la igualdad de oferta, es decir, cuando se brinda a todos los alumnos y alumnas 
un currículo básicamente común que conduce a las mismas metas" (p. 9).

Sin embargo, la equidad en las aulas inclusivas no garantiza la calidad en resultados sino existe compromiso en los responsables del sistema educativo. Para Rambla (2012) "El alcance de la equidad fue ampliado abarcando el acceso, los procesos y los resultados mientras que la calidad se refería a todo aquello que genéricamente tenía que ver con el logro de resultados efectivos de aprendizaje" (p. 34).

Desde el concepto de equidad expuesto por Martín y Rambla, es importante visibilizar con carácter inclusivo a todos los estudiantes, con una oferta educativa que los incluya. De allí que los estudiantes con capacidades y talentos excepcionales se consideran como personas que requieren atención diversificada, a partir del currículo. Para ello, se han explorado diferentes formas del desarrollo de las capacidades y talentos excepcionales, dentro y fuera de la escuela, que incluyen: la aceleración, programas de enriquecimiento, escuelas especializadas, tutorías, grupos de enriquecimiento, diferenciación curricular, entre otros (Dai \& Chen, 2014; Renzulli \& Reis, 2016; Garcia-Cepero \& Proestakis, 2010).

En el contexto escolar, la cuestión tiene que ver con la medida en que los estándares curriculares regulares y la pedagogía coincidan con las necesidades de desarrollo del talento en una etapa o coyuntura específica, y que estas ofertas curriculares y experiencias de aprendizaje garanticen la continuidad del desarrollo del talento en el hogar, la escuela, la comunidad y el ciberespacio.

Las anteriores estrategias, hacen parte del conjunto de oportunidades de desarrollo, que una institución educativa, sola o en conjunto con otras organizaciones, puede ofrecer a sus estudiantes, para responder a las necesidades intelectuales, afectivas y sociales que les permitirán desarrollar su potencial y formarse plenamente como personas. Al respecto Garcia-Cepero \& Proestakis (2010), sugieren que estas estrategias se pueden articular, permitiendo a las instituciones ofrecer un continuo de servicios. Estas estrategias metodológicas que posibilitan el enriquecimiento y potenciación de la excepcionalidad pueden ser adaptadas en el aula escolar a través de su inserción en el currículo.

\section{La aceleración:}

Es una de las estrategias más utilizadas y tiene su origen desde los modelos esencialistas, permitiendo al estudiante avanzar a una velocidad mayor que sus compañeros.

Para Garcia-Cepero \& Proestakis (2010), la aceleración ofrece la posibilidad de adelantarse dentro de los cursos de acuerdo a las características del estudiante. Adicionalmente, es posible hacer procesos de aceleración al interior del aula regular, asignando al estudiante material más avanzado, con el cual puede trabajar de manera independiente a sus compañeros. Sin embargo, aunque esta estrategia se muestra como la práctica más efectiva en términos académicos, de desarrollo social y emocional, no todos los estudiantes se benefician de ella. La aceleración puede ser total o parcial.

\section{Oportunidades de Enriquecimiento:}

¿Qué es el enriquecimiento y cómo fortalece el currículo? Para García-Cepero y González (2004, p. 61) citado por el MEN (2015) "son actividades que permiten al estudiante complementar y desarrollar de manera más amplia y profunda o intensa, contenidos o procesos académicos, culturales y socia- 
les". Uno de los mayores precursores del enriquecimiento del talento es Joseph Renzulli a través del método de enriquecimiento para toda la escuela denomina SEM, soportando en el Modelo Triádico de Enriquecimiento.

Para ello, se pueden establecer dos tipos de enriquecimiento: curricular y extracurricular. El primero permite el trabajo dentro del aula escolar y el segundo en ambientes y contextos externos.

Para Renzulli, después que se identifican los intereses y los estilos de aprendizaje de los estudiantes que presentan capacidades y talentos excepcionales (Cy/oTE, esta sigla se viene utilizando en el documento, ya que se representa para el contexto colombiano) a través de eventos evaluativos como: proyectos, juegos, simulaciones, lecturas, trabajos en equipos, la recitación, el debate, entre otros. Se procede a la compactación y modificación del currículo ordinario, mediante la sustracción de aquellos aprendizajes y contenidos que ya dominan. Así, se posibilita la profundización de áreas de interés del estudiante, que le permitan desarrollar sus capacidades en temas específicos o globales.

\section{Cluster Grouping o grupos de enriquecimiento}

Corresponde a una estrategia de enriquecimiento escolar, el cual reúne o agrupa a los estudiantes reconocidos con talentos o que comparten intereses comunes en un grado o grupo específico. Se reúnen en períodos de tiempo de acuerdo a su horario lectivo para trabajar con un adulto que comparte sus intereses y que tiene un grado de conocimiento y experiencia avanzado.

La división por clúster usualmente se hace con estudiantes talentosos o sobresalientes en más de un área. Sin embargo, se puede hacer dividiendo por especificación del Talento, es decir en un curso se incluirán a todos los estudiantes talentosos en matemáticas y en otro curso los estudiantes talentosos en lenguaje.

Según lo planteado por Gentry M. y Mann, R. (2008) citados por Garcia-Cepero \& Proestakis (2010) los grupos de enriquecimiento generan los siguientes beneficios:

- Desafiar a los estudiantes talentosos al agruparlos en la misma clase, permite la aparición de nuevos talentos en otras clases y cursos.

- Incrementa la habilidad de todos los profesores de conocer las necesidades académicas de sus estudiantes, al reducir el rango de diferencia en los logros académicos de los cursos.

- Los profesores ven a los estudiantes respecto a sus habilidades y logros.

- Extiende los servicios de la educación para talentos a más estudiantes del colegio.

- Ayuda a los profesores a trabajar juntos para planificar currículos diferenciados.

- Ofrece a los estudiantes la oportunidad de desarrollar y crecer recibiendo los servicios educativos, de acuerdo a sus necesidades y capacidades ( $p$. 43).

El modelo de aprendizaje utilizado en los grupos de enriquecimiento, se basa en el enfoque inductivo, centrado en la solución de problemas del mundo real a través del desarrollo de productos y servicios auténticos. Este enfoque es conocido como aprendizaje y enseñanza enriquecidos, permite crear situaciones de aprendizaje que implica el uso de metodologías, desarrolla habilidades de pensamiento de orden superior y aplica de manera auténtica en situaciones creativas. Promueve el aprendizaje cooperativo. 
Currículo por competencias visto desde la educación inclusiva: desafío que responde a los factores de exclusión e inequidad social

\section{Diferenciación y flexibilización curricular}

Desde el enfoque de la educación inclusiva, se vuelve evidente que una de las estrategias para la atención de la diversidad escolar es la implementación de currículos flexibles, que permitan ofrecer diversas oportunidades de crecimiento y desarrollo para todos. Las modificaciones que haga el docente en el currículo juegan un papel importante en el proceso de potenciación y enriquecimiento de las capacidades y talentos excepcionales.

La flexibilización curricular consiste en la diferenciación de los aprendizajes por cada estudiante, teniendo en cuenta sus atributos particulares, intereses, fortalezas y talentos. La diferenciación curricular, sumada a la compactación de currículo y a las unidades de agrupamiento, constituyen una efectiva metodología de trabajo dentro del aula regular. La diferenciación no sólo considera a los estudiantes sobresalientes; también facilita y mejora las estrategias para todo el estudiantado, dependiendo de sus características y habilidades.

\section{CONCLUSIONES}

Para concluir, es importante establecer desde el aula, experiencias educativas encaminadas en forma significativa para todo el alumnado, el cual permita fomentar la transversalidad y la interdisciplinariedad, alejándolos de la preminencia de los contenidos conceptuales presentados aisladamente.

La Educación Inclusiva, corresponde a políticas mundiales de justicia y equidad social, establecidas por la Organización de Naciones Unidas, promulgadas por la UNESCO y ratificadas por los gobiernos, estas reformas se vienen implementando desde hace más de 20 años. El movimiento por la educación inclusiva abarca la totalidad de las necesidades de los educandos, y con más énfasis la de aquellos más vulnerables a la exclusión y marginalización. Es una nueva visión de la educación basada en la diversidad y en el derecho.

Hasta hace poco, la atención destinada a los alumnos con diversidad escolar era mínima, de allí que las reformas educativas no pueden separase de la equidad social para todo tipo de alumnos. Aunque la legislación por sí sola no asegura el éxito de la educación inclusiva es un aspecto muy importante, ya que contar con ella permite establecer derechos y responsabilidades, articular políticas intersectoriales y sectoriales y asegurar la prestación y mantenimiento de recursos y servicios.

Es importante que la legislación que regula los sistemas educativos, contemple la inclusión y la atención a la diversidad como piedra angular, y desarrollar desde los distintos sectores normativas específicas que aseguren el acceso y la adecuada atención a los grupos poblacionales con necesidades educativas y capacidades excepcionales, niños migrantes, niños de la calle, en edad extra escolar, adultos desescolarizados, desplazados, indígenas, raizales, entre otros. Los gobiernos a través de reformas educativas inclusivas son los llamados a diseñar programas específicos orientados a la inclusión de esta población al sistema escolar y para ello implica la revisión de las propuestas curriculares y las estrategias pedagógicas, así como el rediseño de las dinámicas institucionales.

Las estrategias y rediseños curriculares deben propender al desarrollo de las competencias y aprendizajes de los estudiantes, que cobije a todos y todas desde un ambiente escolar. No solamente con el acceso se atiende 
Currículo por competencias visto desde la educación inclusiva: desafío que

a los requerimientos en inclusión, también es necesario la equidad en la prestación, es decir se deriva de los factores en garantizar los procesos educativos y un currículo que responda a sus motivaciones, intereses y necesidades particulares, es decir un currículo diversificado.

Para responder a esta caracterización y la diversidad escolar, se destacan las aulas colaborativas, el lenguaje total, las activida- des directas o mico sociedades, el aprendizaje cooperativo, enfoques temáticos basados en proyectos, el uso de la tecnología y las estrategias de enriquecimiento curricular como la aceleración, los grupos de enriquecimiento, la diferenciación y flexibilización curriculares como métodos de enseñanza que median el aprendizaje y responden a la equidad, desde un planeamiento curricular por competencias visto desde la educación inclusiva.

\section{REFERENCIAS BIBLIOGRÁFICAS}

Castillo, M., \& Gamboa, R. (2017). Desafíos de la educación en la sociedad actual. Revista electrónica diálogos educativo, 12(24), 55-69.

Correa, C. (2009). Currículo, inclusividad y culura de la certificación. Barranquilla: La Mancha del Quijote.

Garcia-Cepero, M. \& Proestakis, M. (2010). Perspectivas de Atención a los Estudiantes con Talento Académico; Una visión global. Centro de Investigación y Desarrollo de Talento DeLTA-UCN, Universidad Católica del Norte, pp. 37-46.

http://www.ficomundyt.org/media/miembros/Perspectivas_de_Atenciona_los_estudiantes_contalento_academico.pdf

Guijosa, C. (5 de julio de 2019). Observatorio de Innovación Educativa.

https://observatorio.tec.mx/edu-news/8-tendencias-de-las-aulas-del-futuro-google-foreducation

Martín, E. (2013). Orientación educativa: atención a la diversidad y educación inclusiva. Madrid, Spain: Ministerio de Educación de España - Editorial GRAÓ, de IRIF, S.L. https:/elibro.net/es/ereader/umecit/49259?page=9.

MEN. (2008). Cartilla 34: Guía de educación inclusiva. Bogotá.

MEN. (2015). Documento de orientaciones técnicas, administrativas y pedagógicas para la atención educativa a estudiantes con capacidades y/o talentos excepcionales en el marco de la educación inclusiva. Santafe de Bogotá.

OCDE. (2019). EducaWeb.

https://www.educaweb.com/noticia/2019/01/23/tendencias-transforman-educacion-18686/ 
Currículo por competencias visto desde la educación inclusiva: desafío que

Osorio F.

responde a los factores de exclusión e inequidad social

Opertti, R. \& Belalcazar, C. (2008). Tendencias de la educación inclusiva a nivel regional e interregional: temas y desafíos. Perspectivas.

https://unesdoc.unesco.org/ark:/48223/pf0000178973_spa

Osorio, F. (2013). De la Integracion a la Inclusión: procesos curriculares para la atención de niños con NEE. Montería.

https://www.edunexos.edu.co/T_grado_Unicordoba/04_COHORTE/OSORIO_F.pdf

Renzulli, J. \& Reis, S. (2016). Enriqueciendo el currículo para todo el alumnado. Madrid: Apeiron ediciones, traducido por Roberto Zans.

SEMANA. (2019). SEMANA.

https://www.semana.com/educacion/articulo/tendencias-que-afectan-la-ensenanza-en-el-mundo/601883

SITEAL. (2019). Inclusión y edquidad Educativa. Buenos Aires: Publicaciones multimedia. https://www.siteal.iiep.unesco.org/eje/inclusion_y_equidad

Stainback, S. \& Stainback, W. (2001). Aulas Inclusivas: Un nuevo modo de enfocar y vivir el currículo. Madrid: Narcea. S.A. de Ediciones.

Rambla, X. (2012). La educación para todos en América Latina: estudios sobre las desigualdades y la agenda política en educación. Buenos Aires, Argentina: Miño y Dávila. Recuperado de https://elibro.net/es/ereader/umecit/42289?page=34

UNESCO. (2015). Declaración de Incheon y Marco de Acción ODS 4 - Educación 2030. Incheon. https://www.gcedclearinghouse.org/sites/default/files/resources/245656s.pdf 